\title{
"Effect of Single Step Adhesives on the Marginal Permeability of Class V Resin Composites - An In Vitro Study"
}

\author{
${ }^{1}$ Dr. Sharanappa Kambale, ${ }^{2}$ Dr Vani hedge, ${ }^{3}$ Dr. Anil Munavalli, \\ ${ }^{4}$ Dr. Sachhi Ramesh, ${ }^{5}$ Dr. Siddhesh Dattatray Bandekar \\ ${ }^{I}$ Sr Lect, Dept of Conservative Dentistry \& Endodontics Vasantdada patil dental college and Hospital \\ Kavalapur Sangli- 416306, India \\ ${ }^{2}$ HOD \& Prof, Dept of Conservative Dentistry \& Endodontics AME'S dental college, Raichur, India \\ ${ }^{3}$ Reader, Dept of Conservative Dentistry \& Endodontics Vasantdada patil dental college and Hospital \\ Kavalapur Sangli- 416306, India \\ ${ }^{4}$ Reader, Dept of Prosthodontic, crown and bridges Vasantdada patil dental college and Hospital Kavalapur \\ Sangli-416306, India \\ ${ }^{5}$ Pg student, Dept of Conservative Dentistry \& Endodontics Vasantdada patil dental college Kavalapur,Sangli
}

\begin{abstract}
:
Aim Of Study: To evaluate the microleakge of self - etch adhesive system at the coronal (enamel) and apical (dentin) margins of class $V$ resin composite restoration.

Methods: Forty eight Class V cavities are prepared [3.0mm X $3.0 \mathrm{~mm} X 1.5 \mathrm{~mm}$ (depth)] on the facial or lingual surface of the non carious tooth with coronal margins in enamel and apical margins in cementum / dentin at the cemento- enamel junction. The preparation will be restored using self - etch adhesive systems (Xeno V, G-Bond, \& clearfil $S^{3}$ Bond) and a control (no adhesive), followed by insertion of Filtek ${ }^{T M}$ P60 resin composite. The teeth were subjected to thermocycled and immersed in $1 \%$ aqueous solution of methylene blue dye for 24 hours. Later sectioned for assessment of microleakage under microscope.

Results: The restored teeth with Xeno V exhibited significantly less leakage than the other adhesive groups in coronal margin, and other group's shows intermediate leakage. The restored teeth treated with clearfil $S^{3}$ exhibited significantly less leakage than other groups in apical margin, and other group's shows intermediate leakage. The control group shows more leakage in both the coronal and apical margins.

Interpretation \& Conclusion: Under the conditions of this in vitro study:

1) At the coronal margin, the preparations treated with Xeno V showed significantly less leakage than the other groups.

2) At the apical margin, the preparations treated with Clearfil $S^{3}$ Bond revealed significantly less leakage than the other groups.

3) At the coronal and apical margin locations, the control group, comprising preparations without treatment using any adhesive system, showed significantly greater leakage than the adhesive groups.

4) Comparing all coronal versus apical surfaces, no significant differences were encountered.
\end{abstract}

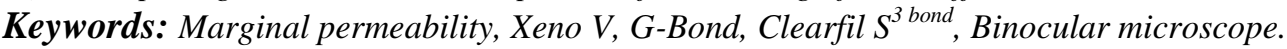

\section{Introduction}

The evolution of dentin bonding agents has progressed rapidly, from adhesion of restorative materials to tooth structure that utilize multiple step procedures, to the development of improved and "easy to use" single component system ${ }^{1}$. Recent dentin adhesives use one of two strategies to interact with dentin smear layer: the Total-etch technique or self- etch technique. Total-etch materials use 30-40\% phosphoric acid to etch dentin and enamel before the clinician applies the adhesive to the preparation. So adhesives, which are being used increasingly, do not require a separate acid-etch step, and do not remove the smear layer. They are composed of aqueous mixtures of acidic functional monomer, generally phosphoric acid esters, with a $\mathrm{pH}$ relatively higher than that of phosphoric acid etching gels. ${ }^{2}$

Self-etching primers were first introduced in Japan. There acidic primers include a phosphonated resin molecule that performs two functions simultaneously- etching and priming of dentin and enamel and are not rinsed off forming a continuum in the substrate and incorporating smear plug into the resin tags. Elimination of rinsing and drying steps reduce the possibilities of over wetting or over drying, which can have a negative influence in adhesion ${ }^{3}$.

Based upon the underlying adhesion strategy, three mechanisms of adhesion are currently in use with modern adhesive system. Those are etch-and-rinse adhesives, self-etch adhesives and glass ionomer adhesives. ${ }^{4}$ One step self-etch adhesives are more commonly associated with lower bonding effectiveness to both enamel/ dentin than are their multi-step counterparts (Bouillagqet et al 2001). Due to their high hydrophilicity, one step- 
etch adhesives behave as semi-permeable membranes, allowing fluids to pass through and seriously jeopardizing bond durability ${ }^{5}$

Adhesion involves some factors. Such as dentin, tooth, patient and material factors. ${ }^{6}$ Self-etch adhesives are widely used, mainly because of their ease- of- use, low technique- sensitivity, and good performance in class $\mathrm{V}$ clinical trials. Besides micromechanical interlocking through hybridization, the potential benefits to additional chemical interaction between the functional monomer and residual hydroxyapatite has regained attention. Specific functional monomer as a part of "mild" two step self-etch adhesives was shown to interact chemically, within a clinically reasonable time, with hydoxyapatite that remain available within the submicron hybrid layer. The specific molecular nature of the functional monomer and the subsequent dissolution rate of its calcium salt have been shown to determine actual chemical bonding efficacy and stability. ${ }^{7}$

Self-etch adhesives may behave as semi permeable membranes that allow the movement of water across the bonded interface and potentially lead to hydrolytic degradation. Because of their high $\mathrm{pH}$, most of the first generation self-etch adhesives resulted in a shallow enamel demineralization compared to that of phosphoric acid. Neverthless, the instrumentation of enamel to remove prismless enamel and / or separate phosphoric acid enamel etching step improved the enamel bonding ability of those self-etch adhesives. ${ }^{8}$

Etch and rinse adhesives are much more micropermeable and affected by pulp fluid compared with self-etching adhesives. Pulp pressure reduces dentin sealing with etch and rinse adhesives, but not with self adhesives. Pulp pressure has no effect on enamel sealing, which is lower when self-etching adhesive is used. ${ }^{9}$ When treating dentin surfaces with futurabond, one-step- self-etch bonding agent, in order to obtain higher micro tensile bond strength, doubling the application time of the adhesive should be considered. ${ }^{10}$ Aim of our study is to evaluate the microleakge of self - etch adhesive system at the coronal (enamel) and apical (dentin) margins of class $\mathrm{V}$ resin composite restorations.

\section{Methodology}

Forty eight extracted non-carious human molars were carefully cleaned of calculus, soft tissue and other debris using a dental curette. The teeth were stored in a $1 \%$ chloramine-T (Fisher Chemical, Fair Lawn. NJ, USA) solution consisting of $12 \%$ active chlorine diluted in distilled water prior to usage.

\section{Cavity Design}

Circular-shaped Class V cavity preparations were cut on the facial or lingual surface at the cementoenamel junction with coronal margins located in enamel and apical margins located in dentin. The preparation-were cut with a \#56 carbide bur in a high-speed hand-piece cooled with an air-water spray. A $45^{\circ}$ bevel was placed on the enamel margin $(0.5 \mathrm{~mm}$ width) using a \#257 diamond bur. Each carbide bur was discarded following preparation of each group of teeth. Preparation dimensions of $3.0 \mathrm{~mm} \times 3.0 \mathrm{~mm} \times 1.5 \mathrm{~mm}$ (depth) were measured with a periodontal probe to maintain uniformity.

\section{Restoration Groups}

The teeth were randomly assigned to four groups $(n=12)$. All materials were used following manufacturer's instructions.

\section{Group 1: Xeno V}

Using a micro brush applicator, Xeno $\mathrm{V}$ was applied to the tooth (enamel and dentin) surfaces and vigorously scrubbed onto the surfaces (15 seconds) for two applications. Excess solvent was removed by gently drying the surfaces with air syringe for at least five seconds. Xeno V was light polymerized for 10 seconds followed by insertion ( 1 increment) of the composite restoration of Filtek ${ }^{\mathrm{TM}} \mathrm{P} 60$.

\section{Group 2: G-Bond}

Using a microbrush applicator, G-Bond was applied to the enamel and dentin surfaces, left undisturbed for 10 seconds and dried thoroughly with an air syringe using maximum pressure. G-bond was light polymerized for 10 seconds followed by insertion of Filtek ${ }^{\mathrm{TM}} \mathrm{P} 60$ composite.

\section{Group 3: Clearfil $\mathbf{S}^{3}$ Bond}

Using a microbrush applicator, Clearfil $\mathrm{S}^{3}$ Bond was applied to the enamel and dentin surfaces for 20 seconds, and then dried thoroughly with an air syringe using maximum pressure for 5-10 seconds. Clearfil $\mathrm{S}^{3}$ Bond was light polymerized for 10 seconds, followed by insertion of Filtek ${ }^{\mathrm{TM}}$ P60 composite

\section{Group 4: Control}

Prior to insertion of Filtek ${ }^{\mathrm{TM}}$ P60 composite into each cavity preparation, no adhesive agent was applied to the enamel and/ dentin surfaces. 
All restorative materials were polymerized with conventional halogen light. The light had previously been monitored with a radiometer and provided adequate intensity $\left(\geq 800 \mathrm{~mW} / \mathrm{cm}^{2}\right)$. The composites were polished with Sof-Lex flexible, aluminum oxide disks of decreasing abrasiveness. The teeth were stored in distilled water at room temperature for 7 days prior to leakage assessment.

\section{Assessment Of Microleakage}

The teeth were thermocycled for 500 cycles in separate water baths of $5^{\circ} \mathrm{C}$ and $55^{\circ} \mathrm{C}$, with a dwell time of 60 seconds in each bath and a transfer time of three seconds. The root apices were sealed with utility wax, and the entire tooth surface was coated with two layers of commercial nail varnish to within $1.0 \mathrm{~mm}$ of the restoration. The teeth were immersed in a $1 \%$ aqueous solution of methylene blue dye for 24 hours at room temperature, thoroughly rinsed to remove excess dye, then invested in clear auto polymerizing resin and labeled. A low-speed diamond saw cooled with water, tooth block was sectioned longitudinally through the center of the restoration from the facial to the lingual surface. Two sections were obtained from each block (24 sections per group), yielding dye penetration (microleakage) readings examined at 40x magnification under binocular microscope. The degree of leakage was determined based on an ordinal ranking system (0-3) as follows:

0 Degree-no leakage

1 Degree-leakage up to one-half the length of the cavity wall

2 Degree-leakage along the full length of the cavity wall, not including the axial surface

3 Degree-leakage along the full length of the cavity wall, including the axial surface.

\section{Results}

A mean difference value of degree of leakage by Xeno V (mean value- 0.67) is statistically highly significant when compared to other groups (mean values: G-Bond-1.6, Clearfil S3 Bond- 1.3, and control group3.0) at coronal region. In apical region Clearfil S3 Bond(mean value-0.7) is statistically highly significant when compared to other groups (mean values: G-Bond-2.0, Xeno V-2.38, and control group- 3.0) ( Summarized in Table 1)

Inter group comparison of degree of leakage of single step adhesives (Xeno-V, G-Bond, and Clearfil S3 Bond). Wilcoxon Signed rank test is used and $\mathrm{p}<0.001$ which is highly significant.

\begin{tabular}{|c|c|c|c|c|c|}
\multicolumn{2}{c}{ Table. 1: Comparison between Coronal and Apical region Mean Values } \\
\hline Study Groups & Coronal & Apical & $\begin{array}{c}\text { Mean } \\
\text { Diiference }\end{array}$ & P* Value & Significance \\
\hline Xeno-V & 0.67 & 2.38 & 1.71 & $\mathrm{P}<0.001$ & $\mathrm{HS}$ \\
\hline G-Bond & 1.6 & 2.0 & 0.38 & $\mathrm{P}<0.01$ & $\mathrm{~S}$ \\
\hline Clearfil S ${ }^{3}$ Bond & 1.3 & 0.7 & 0.54 & $\mathrm{P}<0.01$ & $\mathrm{~S}$ \\
\hline Control & 3.0 & 3.0 & 0.00 & $\mathrm{P}>0.05$ & $\mathrm{NS}$ \\
\hline
\end{tabular}

* Wilcoxon Signed rank test

Pairwise comparison of all groups for marginal permeability at both coronal and apical region, using Kruskall Wallis test and Mann Whitney U test. In coronal region Xeno V had statistically high significant difference and shows less leakage compared with G-Bond, Clearfil S ${ }^{3}$ Bond and control groups. In apical region clearfil S3 Bond had statically high significant difference and shows less leakage compared with G-Bond, Xeno V and control groups. ( Summarized in Table $2 \& 3$ )

Table.2: Comparison among study groups in coronal region

\begin{tabular}{|c|c|c|c|c|c|c|}
\hline Coronal & & & & & ficant Pairs** & \\
\hline Study Groups & Mean & $\begin{array}{l}\text { P* Value, } \\
\text { sig }\end{array}$ & Xeno-V & G-Bond & Clearfil $\mathrm{S}^{3}$ Bond & Control \\
\hline Xeno-V & 0.67 & \multirow{4}{*}{$\mathrm{P}<0.001 \mathrm{HS}$} & - & $\mathrm{P}<0.001 \mathrm{HS}$ & $\mathrm{P}<0.05 \mathrm{~S}$ & $\mathrm{P}<0.001 \mathrm{HS}$ \\
\hline G-Bond & 1.6 & & - & - & $\mathrm{P}>0.05 \mathrm{NS}$ & $\mathrm{P}<0.001 \mathrm{HS}$ \\
\hline Clearfil $\mathrm{S}^{3}$ Bond & 1.3 & & - & - & - & $\mathrm{P}<0.001 \mathrm{HS}$ \\
\hline Control & 3.0 & & - & - & - & - \\
\hline
\end{tabular}

* Kruskall Wallis test 
** Mann Whitney U test

Table.3: Comparison among study groups in apical region

\begin{tabular}{|c|c|c|c|c|c|c|}
\hline Apical & & & & & cant Pairs** & \\
\hline Study Groups & Mean & $\mathrm{P}^{*}$ Value, sig & Xeno-V & G-Bond & Clearfil S ${ }^{3}$ Bond & Control \\
\hline Xeno-V & 2.38 & \multirow{4}{*}{$\mathrm{P}<0.001 \mathrm{HS}$} & - & $\mathrm{P}>0.05 \mathrm{NS}$ & $\mathrm{P}<0.001 \mathrm{HS}$ & $\mathrm{P}>0.05 \mathrm{NS}$ \\
\hline G-Bond & 2.0 & & - & - & $\mathrm{P}<0.001 \mathrm{HS}$ & $\mathrm{P}<0.05 \mathrm{~S}$ \\
\hline Clearfil $\mathrm{S}^{3}$ Bond & 0.7 & & - & - & - & $\mathrm{P}<0.001 \mathrm{HS}$ \\
\hline Control & 3.0 & & - & - & - & - \\
\hline
\end{tabular}

* Kruskall Wallis test

*** Mann Whitney Utest

\section{Discussion}

This study evaluated the microleakage of four single component seventh generation self-etch adhesives, all of which demonstrated dye penetration (leakage) at both the coronal and apical margins. The control in this study was a "no adhesive" group containing no adhesive system application. This protocol was considered an appropriate step in that a previous study by Owens, Johnson and Harris tested micro leakage using separate self-etch (seventh generation) and total-etch systems. ${ }^{39}$ Not wishing to duplicate protocol of that study, the use of a "no adhesive" was therefore adopted as a "negative control." ${ }^{1}$

SE adhesives are less technique sensitive than are Total-etch adhesives. Self-etch adhesives do not remove the smear layer from dentin completely, so clinicians believe they cause less post operative sensitivity than do Total-etch adhesives. Furthermore, Self-etch adhesives are not likely to results in a discrepancy between the depth of demineralization and the depth of resin infiltration, because both processes occur simultaneously. Another advantage of SE adhesives is that moist bonding is not required. ${ }^{2}$

The restorations treated with the Xeno V and Clearfil S3 Bond adhesive systems revealed significantly reduced leakage at the coronal margins compared to the other adhesives, with Clearfil S3 Bond showing significantly less leakage at the apical margins. Also, at the apical margin, Xeno V showed significantly greater leakage than the other groups, except the control. Overall, Clearfil S3 Bond revealed superior (not necessarily always significant) results at both margin locations. Explanations for these results include the type of solvent used in the adhesive systems. Instead of acetone, Clearfil S3 Bond utilizes alcohol as the primer component solvent or "drying agent" for dentin surface conditioning prior to adhesive component attachment. ${ }^{1}$

The Clearfil $\mathrm{S}^{3}$ Bond formulation includes a proprietary "Molecular Dispersion Technology," enabling a two-phase liquid, hydrophilic/hydrophobic component homogenous state at the molecular level, reportedly resulting in reduction and/ or loss of water droplets at the adhesive interface and therein a superior bond. ${ }^{11}$

Also the 10-Methacryloyloxydecyl dihydrogen phosphate (MDP) adhesive monomer molecular structure allows for decalcification and penetration into tooth structure, "creating a chemical bond to calcium." This molecular formulation is purposely beneficial when bonding to enamel surface substructure; whereby, MDP chemically bonds to hydroxyl apatite, as opposed to a micro-mechanical bond created using total-etch systems (phosphoric acid). ${ }^{11}$ In this study, although Clearfil $\mathrm{S}^{3}$ Bond's performance was good overall, the previously stated claims were not completely justified.

GB are not result in a defined etching pattern on unground enamel; whereas on ground enamel both resulted in islands of superficially dissolved enamel within areas without evidence of enamel dissolution. This was somewhat predictable, as other authors have reported that GB result in severe enamel micro leakage and debonding following thermal stresses and may be unable to penetrate through smear layers. ${ }^{12,13,14}$ The behavior of GB may be a result of the presence of 4-META, which does not bond very strongly to hydroxyapatite. ${ }^{15}$

But, the functional monomer 4- acryloxyethyltrimellitic acid (4-AET) has been shown to interact with $\mathrm{Ca}^{2+}$ from apatite crystallites within the partially demineralized hybrid layer to form an insoluble calcium salt (4-AETCa) that may aid in bonding this resin system to dentin. 4-AET has also been shown to bond chemically to both dentin apatite and collagen. A chemical interaction between hydroxyapatite and functional monomers in the adhesive leads to higher bond strengths than the adhesives that rely only on micromechanical retention to dentin substrate. ${ }^{13}$

Interestingly, when Xeno was used, the thickness of the hybrid layer was greater under pulp pressure conditions. A previous study reported that a higher water concentration improved acidic monomer ionization; with a consequent increase in thickness of the hybrid layer. ${ }^{16}$ Extra water from the pulp would increase the water concentration and acidic monomer ionization and, consequently, the etching depth. 
Clinical research conducted by Brackett and others ${ }^{17}$ concluded that single component self-etch adhesives performed poorly, with a retention rate of only $50 \%$ to $55 \%$ after 18 months. In an intra-individual patient-based clinical comparison study, the results revealed that self-etch adhesives showed a faster progressive marginal degradation in non-carious cervical lesions. ${ }^{18}$ Also, in a systematic review of current clinical trials involving dental adhesive systems, the results showed that "three-step etch-and-rinse adhesives and two-step self-etch adhesives showed a clinically reliable and predictably good clinical performance and the clinical effectiveness of two-step etch-and rinse adhesives was less favorable, while an inefficient clinical performance was noted for the one-step self-etch adhesives."19

Two self-etch adhesives may result in better enamel marginal sealing than all-in-one self-etch adhesives. ${ }^{20}$ The etch-and-rinse and self-etch adhesives systems can be used with confidence; however, SE adhesives showed a faster and more progressive enamel marginal degradation ${ }^{21}$.

The specific nature of restoration failure (microleakage) for each adhesive system is unknown, although several factors were strongly suspected: inefficiency of acidic monomers in alteration of the smear layer for classic hybrid layer formation, cavity C-factor, orientation of dentinal tubules/enamel rods to the cementoenamel junction, use of acetone-based solvent primer systems and post-treatment stresses caused by polymerization contraction.

The results from this study demonstrate that the "dynamic" nature of the dentin substrate morphology is indeed an important factor and possibly an insurmountable impediment for perfect adhesion of restorative materials to tooth structure.

Under the conditions of this in vitro study:

\section{Conclusion}

1. The clearfil $\mathrm{S}^{3}$ bond showed less restoration leakage compared to the other self-etch adhesives at both enamel and dentin margins.

2. At the coronal margin, the preparations treated with Xeno V showed significantly less leakage than the other groups

3. At the apical margin, the preparations treated with Clearfil $\mathrm{S}^{3}$ Bond revealed significantly less leakage than the other groups.

4. At both the coronal and apical margin locations, the control group, comprising preparations without treatment using any adhesive system, showed significantly greater leakage than the adhesive groups

\section{References}

[1]. Owens BM, jonson WW. Effect of single step adhesives on the marginal permeability of class - V resin composites. Oper dent 2007; 31(2):67-72.

[2]. Perdigao J, Geraldeli S \& Hodges JS. Total-etch versus self-etch adhesives: effect on post operative sensitivity.Jounal of American dental association 2003; 134(12): 1621-1629.

[3]. Munck JD, van landyut K, peuman SM, Poitevin A, Lanbrechts P, Braem M, van meerbeck. A critical review of the durability of adhesive to tooth tissue: Methods \& Results. J dent res 2005; 84(5); 118-132.

[4]. K L Van Landuyt, J De Munck, J Snauwaert, E Coutinho, A Poitevin, Y Yoshida, S Inoue, M Peumans, and Vn Meerbeek. Monomer - solvent phase separation in One- step self-etch adhesives. J dent res 2005; 84(2): 183-188.

[5]. Herald O heyman, Stephen c Bayne. Current concepts in dentin bonding. Jou of American association 1993; 124:27-36.

[6]. S Inoue, K Koshiro, Y Yoshida, J Demunck, K Nagakane, K Suzuki, h Sano, and B Van Meerbeek. Hydrolic stability of self-etch adhesives bonded to dentin. J dent res 2005; 84(12): 1160-1164.

[7]. K L Van Landuyt, J De Munck, J Snauwaert, E Coutinho, A Poitevin, Y Yoshida, S Inoue, M Peumans, and Vn Meerbeek. Monomer - solvent phase separation in One- step self-etch adhesives. J dent res 2005; 84(2): 183-188.

[8]. J Perdigao, G Gomes. In Vitro bonding perpormance of self-etch adhesive: II- Ultramorphological evaluation. Oper dent 2008; 33(5): 534-549.

[9]. JI Rosales-Leal, FJ de la Torre-Moreno, M Bravo. Effect of pulp pressure on the micropermiability and sealing ability of etch and rinse and self-etching adhesives. Oper dent 2007; 32(3): 242-250.

[10]. M Toledano, JP Proeca, MCG Erhardt, E Osorio, FS Aguilera, R Osorio, FR Tay. Increased in dentin -bond strength if doubling application time of an acetone- containing one-step adhesive. Oper Dent 2007; 32(2): 133-137.

[11]. Clearfil s3 bond product information Kurary dental (2005) 1-4.

[12]. Pashley EL. Agee KA, Pashley DH \& Tay FR. Effects of one versus two applications of an unfilled, all-in adhesive on dentine bonding. Jou of Dent 2002; 30(3) 83-90.

[13]. M Ikeda, K Tsubota, T Takamizawa, T Yoshida, M Miyazaki, JA Platt. Bonding durability of single- step adhesives to previously acid- etched dentin. Oper dent 2008; 33( 6): 702-709.

[14]. Santini A, Ivanovic V, Ibbetson R \& Milia E (2004) Influence of cavity configuration on microleakage around Class V restorations bonded with seven self-etching adhesives Journal of Esthetic and Restorative Dentistry; 16(2): 128-135.

[15]. Yoshida Y, Nagakane K Fukuda R, Nakayama Y, Okaz; V. Shintani H, Inoue S, Tagawa Y, Suzuki K, De Munck J \& Van Meerbeek B. Comparative study on adhesive performance of functional monomers Jou of Den Res 2004;83: 454-45.

[16]. Hiraishi N, Nishiyama N, Ikemura K, Yan JY, King NM, Tagami J, Pashley DH \& Tay FR. Water concentration in self etching primer affects their aggressiveness and bonding efficacy to dentin.J den research2005; 84(7):653-658.

[17]. WW Brackett, MG Brackett, A Dib, G Franco, H Estudillo. Eighteen-month Clinical Performance of a Self-etching Primer in Unprepared Class V Resin Restorations. Operative Dentistry 2005, 30-4:424-429 
[18]. Dalton BD, Ezecelevski IG, Reis A, Van Dijken JW \& Locruercio AD. An 18-month's evaluation of self-etch and etch \& rinse adhesive in non-carious cervical lesions Acta Odontological Scandinavia2005; 63(3):173-178.

[19]. Peumans M, Kanumilli P, De Munck J, Van Landuyt K, Lambrechts P \& Van Meerbeek B. Clinical effectiveness of contemporary adhesives: A systematic review of current clinical trials Dental Materials 2005;21(9):864-881.

[20]. Jorge Perdigao, Paulo Monteine, George Gomes. In vitro enamel sealing of self-etch adhesives. American association for dental research,2009;40(3): 225-233

[21]. Alessandro, Dax Dalton, Luiz Narciso, Alessandra Reis. A 36- month evaluation of self- etch and etch-and-rinse adhesives in noncarious cesvical lesions. JADA 2007; 138:507-514. 